\title{
Pathophysiologic Enigma of COVID-19 Pandemic with Clinical Correlates
}

\section{IJCRR}

Section: Healthcare

Sci. Journal Impact

Factor: $6.1(2018)$

ICV: $90.90(2018)$

Scopus'

\section{Sana Parveen ${ }^{1}$, Shraddha Jain ${ }^{2}$}

'MBBS, Post Graduate Resident, Department of Otorhinolaryngology and Head Neck Surgery, Jawahar Lal Nehru Medical College, Datta Meghe Institute of Medical Sciences (Deemed to be University), Sawangi (M), Wardha, Maharashtra, India-442004; ${ }^{2}$ MBBS, MS(ENT), PhD, Professor, Department of Otorhinolaryngology and Head Neck Surgery, Jawahar Lal Nehru Medical College, Datta Meghe Institute of Medical Sciences (Deemed to be University), Sawangi (M), Wardha, Maharashtra, lndia-442004.

\section{ABSTRACT}

Introduction: This review article examines the virology, modes of transmission, risk factors, clinical course, diagnosis and treatment of Coronavirus disease based on the various studies done in the past 8 months.

Background: Covid-19(Coronavirus disease 2019) has been named as a novel virus as there has been no known cases of infection with this strain in human beings. Origin of this virus is alleged to be from a family of bats and pangolins, in Wuhan, China in Dec 2019 from a seafood market, and since then the spread of this virus has been unstoppable.After a single introduction into the human population, the Chinese authorities immediately notified the WHO about the increasing number of COVID -19 cases. An epidemic was caused due to the SARS virus in China in 2003, and hence this virus has been renamed as SARS-Cov-2 because of its close relation to it. The symptoms of this disease can vary from mild flu-like to severe respiratory distress requiring admission to ICU.

Methods: The literature on COVID-19 was retrieved from PubMed, Google Scholar and Cochrane database. Keywords and phrases used during the search included "Covid-19," "pandemic," "Novel" and "Coronavirus".

Conclusion: At present, no definitive treatment is available for this virus; only symptomatic management is undertaken. Further research is being undertaken to develop the Coronavirus vaccine and the use of plasma of treated patients to manage active infected cases.

Key Words: COVID, Pandemic, Seafood, Recombination, Zoonotic spillover

\section{INTRODUCTION}

The virus has been named so attributing to its crown like appearance under microscopy. Coronaviruses are positive sense single stranded RNA enveloped viruses. ${ }^{1}$ Till now, four genera namely - a, b, g, d, have been identified. The novel-b Coronavirus shows maximum similarity to the genomic sequence of two bat-derived (SARS)-like coronaviruses. This virus was then named "SARS-CoV-2" by theInternational Virus Classification Commission on February 11, 2020 also known as COVID-19 - acronym for coronavirus disease. The spread of the virus began in December 2020 and since then, it has spread to more than 203 countries, infecting more than 10 million people and causing mortality in several nations. Apart from this, there have been two more epidemics in the past two decades due to coronaviruses namely, SARS- severe acute respiratory syndrome and MERS- Middle East respiratory syndrome. ${ }^{2}$

\section{The phenomenon of "Zoonotic Spillover" and "Recombination"}

The most probable site of isolation of the virus are the seafood markets of Wuhan, China. Wild animals over here are sold as pets and delicacies or kept in captivity. Animals and human beings are kept in close contact with each other in this area, due to which a disastrous mixture of various species and viruses can occur. This has led to the phenomenon known as "Zoonotic Spillover". ${ }^{3}$

Two mechanisms namely antigenic drift and shift, have been implicated in giving rise to the pandemic status of the novel Coronavirus. Antigenic drift is when many small mutations

\section{Corresponding Author:}

Dr. Shraddha Jain, MBBS, MS(ENT), PhD, Department of Otorhinolaryngology and Head and Neck Surgery, Jawahar Lal Nehru Medical College, Datta Meghe Institute of Medical Sciences (Deemed to be University), Sawangi (M), Wardha, Maharashtra-442004, India. Mobile: 9881836366; E-mail: sjain_med@yahoo.co.in

ISSN: 2231-2196 (Print)

Received: 12.05 .2020
ISSN: $0975-5241$ (Online)

Revised: 11.06 .2020
Accepted: 20.06 .2020
Accepted: 05.07 .2020 
occur and accumulate over time to give rise to different strains of a virus. Many scientists suggest that the SARSCov-2 virus is formed as a result of a recombination between two viruses, one which is close to the bat virus and the other similar to the pangolin virus. It is a chimera between two preexisting viruses, also known as antigenic shift, which is also seen in many influenza viruses. Various studies have already explained this recombination mechanism. ${ }^{4}$ Recombination is the process by which a new virus develops which results in infection of a new host species. For this to occur, the two different viruses should infect the same organism at the same time.

\section{Pathogenesis-}

The most commonly identified route of entry of the virus into the human body is through the nostrils i.e. aerosol borne ; second one being fomites i.e. surfaces that frequently coming in contact with human skin which can transmit the infection to another person and hence the transmission rate is very high for this infection. The most important predictor of virus entry into host cells has been reported to be Coronavirus $\mathrm{S}$ Protein. There is direct fusion between the virus membrane and the cell plasma membrane by which there is entry of the virus.

New envelope glycoproteins are formed and inserted into the ER or Golgi apparatus, and the formation of nucleocapsid takes place by the combination of nucleocapsid protein and genomic RNA. Then germination of viral particles occurs and vesicles are formed which fuse with cell membrane to release the viral particles. ${ }^{5}$

\section{Antigen presentation in coronavirus infection}

Upon entry of the virus in the cells, its antigen is presented to the antigen presentation cells(APC) which is done by major histocompatibility complex (MHC; or human leukocyte antigen(HLA) in humans) and then identified by virus-specific cytotoxic $\mathrm{T}$ lymphocytes (CTLs). The antigen presentation of SARS-CoV mainly depends on MHC I molecules, but studies have shown that MHC II also contributes to its presentation.

\section{Humoral and cellular immunity}

Like common viral infections, the antibodies against SARS$\mathrm{CoV}$ virus have a typical IgM and IgG production. At the end of 12 weeks, the S-specific IgM antibodies disappear, while the IgG antibodies can last for a longer time. The antibodies produced are primarily S-specific and N-specific. Various reports suggest that the number of CD4p and CD8p T cells in the blood of SARS-CoV-2-infected patients is reduced. ${ }^{5}$ which is also seen in the acute phase response. Studies have shown that even in the absence of an antigen, CD4p and $\mathrm{CD} 8 \mathrm{p}$ memory $\mathrm{T}$ cells can persist for four years in a part of SARS-CoV recovered individuals and can perform $\mathrm{T}$ cell proliferation. $^{5}$

\section{Cytokine storm in COVID-19}

ARDS is the commonest end event for all three identified Coronaviruses namely; SARS-CoV-2, SARS-CoV and MERS-CoV infections. IFN-g, IFN-a, TNF-a, and TGF b are pro-inflammatory cytokines, which, along with CCL2, CCL3, and CCL5, are chemokines released in a huge quantity by macrophages, monocytes, lymphocytes and platelets in this infection. This gives rise to a severe uncontrolled inflammatory response, known as cytokine storm. The immune system violently attacks the body, which many studies have suggested that is supposedly an allergic response due to the chemokines. It, in turn, can cause multiple organ failure, and ultimately lead to death. ${ }^{5}$

\section{Evasion of immune system}

Many strategies are used by Coronavirus to ensure immune evasion and escape destruction by the host cells. Modification of pattern recognition receptors and the ability to affect antigen presentation are ways by which they avoid detection by the antigen presenting cells. Studies have also shown that gene expression related to antigen presentation can also be down-regulated by the MERS$\mathrm{CoV}$ infection.

\section{Virus structural protein binding porphyrin-}

Recent studies have shown that the virus has few structural proteins which can bind the porphyrin in heme and reduce the oxygen binding capacity of haemoglobin, ultimately leading to hypoxia. This can further give rise to cardiac as well as neurological symptoms in the patient like- stroke, decreased consciousness, myopathy and loss of sense of smell or taste. ${ }^{5,6}$

\section{Clinical features}

The clinical manifestations of this disease range from being asymptomatic to rapidly progressing disease. It has been noted that most of the cases are mild to moderate and around $4 \%$ of the cases only progress to fulminant disease, suggesting that the mortality rate is much lower than MERS and SARS infections. Symptoms begin with a fever, cough, sore throat, nasal discharge and obstruction along with myalgia. Rarely a patient can present with headache and diarrhoea as the only symptom as some studies have suggested. About $50 \%$ of the patients had dyspnoea (median duration- 8 days). Lymphocytopenia was seen in $63 \%$ of patients. ${ }^{7}$ Complications like acute respiratory distress syndrome, secondary infections and acute heart injury were also present. Few of the patients required to be treated in the ICU with ventilator support. The median incubation period was 14 days, which is also the suggested quarantine period for any infected patient. Studies have shown that 
among confirmed cases, maximum were aged 30-79 yearsand had mild/moderate pneumonia. ${ }^{8}$

\section{Diagnosis}

\section{Physical examination}

The infected patients have symptoms like dry cough, difficulty in breathing, fever, nasal congestion, runny nose or other upper respiratory tract symptoms. Recent researches have also shown that the disease can also present with anosmia, hyposmia and dysgeusia .

\section{Imaging}

Chest X-ray: In mild cases, chest X-Ray is suggestive of many small patches and interstitial changes, remarkable in the lung periphery. Ground-glass opacities, shadows with or without pleural effusion, can be present in high risk cases.CT scan of the thorax of the infected patients was suggestive of - ground-glass opacity and consolidation in both lungs, especially in the lung periphery. Multiple lobar lesions can be present in both lungs in children with severe infection. ${ }^{10}$

\section{Laboratory diagnosis}

The definitive diagnostic modality is collection of nasopharyngeal and oropharyngeal swabs sent for RT-PCR testing followed by subsequent isolation of the suspected patient. ENT specialists usually take the test and they should be standing on the side of the patient while enduring the swab to prevent direct aerosol contact from the patient. The person receiving the sample must be wearing complete Personal Protective Equipment. ${ }^{11}$

To collect the oropharyngeal sample, the swab is moved along the back of the throat as well as bilateral tonsillar beds. In the nose also the same swab is used in order to conserve swabs, below the inferior turbinates up to the nasopharynx. These samples contain high viral loads, and hence should be used for testing wherever possible. In some cases, endotracheal aspirate, broncheoalveolar lavage can also be taken, where lower respiratory samples are needed. Currently, the time taken for the results is around 48 hours. Various labs have been developed to give the results in fastest time, namely -Neuberg Diagnostics, Oncquest, Mylabs, which conduct the test by Rapid Diagnostic Test Kits (RDT), which gives the test results in 45 minutes. Till then the patient is kept in isolation in order to prevent the spread of the infection in case the patient turns out to be positive. ${ }^{12}$

\section{Treatment}

Various guidelines have been given by the WHO and the CDC for managing the patient.

A COVID suspect is defined as any person with

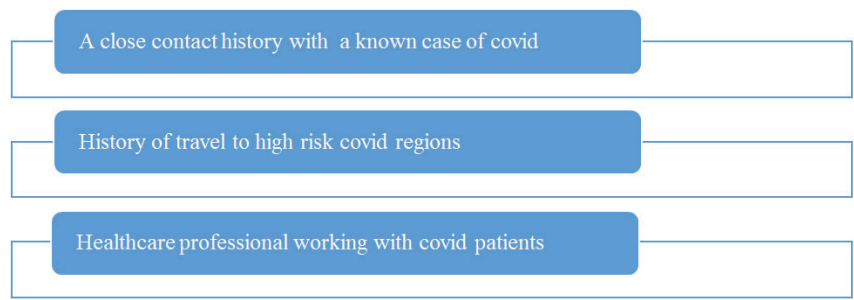

Figure 1: Definition of a COVID suspect patient.

A study published in "The Lancet" states that the lesser severity of coronavirus impact may be linked to national policies on BCG childhood vaccination, while citing the examples of multiple randomised control trials conducted in Netherlands and Australia ${ }^{12}$. Currently, no specific drug or vaccine is available for this viral infection. Only symptomatic treatment consisting of anti-allergics, antipyretics, rest and isolation is advisable. ${ }^{13}$

Ongoing research is suggestive of the presence of an mRNA sequence found in Southeast Asian population, which has been known to be protective of this virus. Hence, the reason why not very severe cases of pneumonia or ARDs have been reported in this population. Many US scientists have suggested that the Bacillus Calmette-Guerin (BCG) vaccine, administered to Indian children soon after birth in an attempt to protect against tuberculosis, could be a "game-changer" in the fight against the deadly coronavirus. ${ }^{14}$

Chloroquine- an antimalarial has been implicated in reducing the severity of the acute immune response by the virus, but further research is required in order to identify the riskbenefit ratio as some cases of death due to Q-T prolongation followed by sudden cardiac arrest have been reported after some doctors took the drug as a prophylactic. ${ }^{15}$ Few scientists have found that immunosuppressive drugs like Tacrolimus and Prednisolone can help reduce the immune response and prevent the patient from developing a strong reaction to the virus, as seen in patients of solid organ transplantation affected with COVID-19. ${ }^{16}$

Also, further studies are being conducted to isolate the plasma of treated COVID patients, which can be used to treat active symptomatic patients. Numerous clinical trials are ongoing in Wuhan, Maryland and other places, to develop a vaccine for this pandemic causing virus..$^{17,18}$

\section{Measures to control and prevent transmission of infection}

As advertised by the WHO and the government authorities of multiple countries, two main steps are extremely crucial to control the spread of this virus-

Social distancing and handwashing.

Social distancing means maintaining at least a distance of 6 feet from other people and handwashing practiced for at 
least 20 seconds and with all the proper steps. This has been achieved by stringent measures like a two month lockdown in India as well as China, closure of schools, colleges, any sort of public gatherings, movie halls etc. along with strict action taken by the government if these rules are not obeyed. ${ }^{19}$ Since exact treatment is not known yet, the best way to be protected from this virus is by preventing it, researchers say. For healthcare providers of known /suspected COVID patientsit is essential to wear personal protective equipment and follow proper guidelines for putting on and taking off the PPE.

\section{Areas of further research}

It is important to determine in which organism did the recombination occurs, (pangolin / bat/another species) and under which environmental conditions it took place.

Drugs can be developed to target the S protein or inhibit the virus's entry into the human cell. Along with that destroying the immune evasion mechanism of this virus is crucial in its specific drug development. ${ }^{20}$

The plasma of COVID treated patients can be isolated and used in the treatment of actively infected cases; further studies need to be done in this area. Also by studying the T-cell responses in patients, development of vaccines can be done, which imperative during this time.

\section{DISCUSSION}

The Coronavirus has emerged as an extremely virulent pandemic, causing disruption of lives of thousands of individuals all over the world. Because of the lockdown which has been imposed in different parts of the world, a significant amount of mortality has been prevented, especially in developing countries like India. The coming two weeks will be quite crucial for this country as the incubation period will be over and due to the relaxation in the lockdown, symptomatic patients will start coming in.

\section{CONCLUSION}

The onset of this new disease has cost us almost 2 lakh lives and as on May 9, 2020, there are more than 33 lakh cases, out of which around 5 lakh will require ventilatory support in the coming weeks. With a sudden shock to the healthcare sector, there has been a tremendous loss to the economy, finances of the nations, loss of wages to the daily wagers, and business shutdown. If managed in an organised way, there's a way out of this viral situation.

\section{ACKNOWLEDGEMENT}

We acknowledge the support of our institution Datta Meghe Institute of Medical Sciences; and the Department of Research and Development of our university for all the help lent in preparing and submitting this manuscript.

Sources of funding - None.

Conflicts of interest - Nil

\section{REFERENCES}

1. Remuzzi A., Remuzzi G. COVID-19 and Italy: what next?', Health Policy. Published Online. March 12, 2020. https://doi.org/10.1016/S0140-6736(20)30627-9

2. Zhou F., Yu T., Du R., Fan G., Liu Y., et al. ; Clinical course and risk factors for mortality of adult inpatients with COVID-19 in Wuhan, China: a retrospective cohort study. Lancet 2020; 395: 1054-62 2020

3. Decaro N., Lorusso A. et al.; Novel human coronavirus (SARSCoV-2): A lesson from animal coronaviruses Vetinerary Microbiology. 2020 May; 244: 108693. Published online $2020 \mathrm{Apr}$ 14. doi: $10.1016 /$ j.vetmic.2020.108693

4. Graham R.L, Baric S. R. Recombination, Reservoirs, and the Modular Spike: Mechanisms of Coronavirus Cross-Species Transmission. Journal of Virology Mar 2010, 84 (7) 3134-3146;

5. Liu W., Li H . COVID-19: Attacks the 1-Beta Chain of Hemoglobin and Captures the Porphyrin to Inhibit Human Heme Metabolism. posted online on chemrxiv.com

6. Green A.J., Josephson S. A The Spectrum of Neurologic Disease in the Severe Acute Respiratory Syndrome Coronavirus 2 Pandemic Infection Neurologists Move to the Frontline.JAMA Neurol. Published online April 10, 2020. doi:10.1001/jamaneurol.2020.1065

7. Pambuccian S.E. The COVID-19 pandemic: Implications for the cytology laboratory. Journal of the American Society of Cytopathology 2020; March 232020 https://doi.org/10.1016/j. jasc.2020.03.001

8. Verity R., Okell L.C., Dorigatti I., Winskill P .,Estimates of the severity of coronavirus disease 2019: a model-based analysis Lancet Infect Dis 2020 March 30, 2020. https://doi.org/10.1016/ S1473-3099(20)30243-7

9. Kaye R, Chang D, Kenzhaya K, COVID-19 Anosmia Reporting Tool: Initial Findings. American Journal of Otolaryngology and Head and Neck surgery. April 28, 2020. Published online. https://doi.org/10.1177/019459982092299

10. Adhikari S.P., Mengh S., Wu Y. et al.., Epidemiology, causes, clinical manifestation and diagnosis, prevention and control of Coronavirus disease (COVID-19) during the early outbreak period: a scoping review. Infectious Diseases of Poverty volume 9, Article number: 29 March 17 (2020). https://doi.org/10.1186/ s40249-020-00646-x

11. Leitmeyer K., Zambon M., et al. Laboratory testing for coronavirus disease (COVID-19) in suspected human cases. WHO Interim Guidance. Published online on March 19, 2020.

12. Curtis N., Sparrow A., et al., Considering BCG Vaccination to lesser the impact of COVID-19. The Lancet. Volume 395, p1545-1546. May 16, 2020.

13. Chavez S., Long B., Koyfman A. et al., Coronavirus Disease (COVID-19): A primer for emergency physicians, American Journal of Emergency Medicine (March 22 2020), https://doi. org/10.1016/j.ajem.2020.03.036

14. X. Li et al., Molecular immune pathogenesis and diagnosis of COVID-19, Journal of Pharmaceutical Analysis, https://doi. org/10.1016/j.jpha.2020.03.001 
15. Moore N., Chloroquine for COVID-19 Infection. Nature Public Health Emergency Collection. 2020 April 7. p 1-2. doi: 10.1007/ s40264-020-00933-4 [Epub ahead of print] .

16. Zhang Z., Zhang Q., Clinical Characteristics and Immunosuppressant Managemnt of Coronavirus Disease 2019 in Solid Organ Transplant Recipients. American Journal of Transplantation. 2020 April 13. doi: 10.1111/ajt.15928. [Online ahead of print ]

17. "Safety and Immunogenicity Study of 2019-nCoV Vaccine (mRNA-1273) for Prophylaxis SARS CoV-2 Infection - Full Text View - ClinicalTrials.gov". clinicaltrials.gov. US National Library of Medicine, National Institutes of Health. Retrieved March 312020.
18. Mak E., China approves first homegrown COVID-19 vaccine to enter clinical trials. BioWorld. Retrieved March 242020.

19. Rodriguez-Morales A.J., et al., Clinical, laboratory and imaging features of COVID-19: A systematic review and meta-analysis. Travel Medicine and Infectious Disease, March 13, 2020. https://doi.org/10.1016/j.tmaid.2020.10162

20. Prem K, Liu Y Russell T.W , Kucharski A.J., The effect of control strategies to reduce social mixing on outcomes of the COVID-19 epidemic in Wuhan, China: A modelling study; Lancet Public Health. March 25, 2020. https://doi.org/10.1016/S24682667(20)30073-6. 